\title{
Fluidification of Stochastic Petri Net by Non Linear Timed Continuous Petri Net
}

\author{
Nabil El Akchioui \\ Faculty of Science and Technology, University the First Mohamed, Oujda, Morocco \\ Email address: \\ nabil.elakchioui@yahoo.fr \\ To cite this article: \\ Nabil El Akchioui. Fluidification of Stochastic Petri Net by Non Linear Timed Continuous Petri Net. American Journal of Embedded \\ Systems and Applications. Vol. 5, No. 4, 2017, pp. 29-34. doi: 10.11648/j.ajesa.20170504.11
}

Received: November 6, 2017; Accepted: November 16, 2017; Published: January 2, 2018

\begin{abstract}
Reliability analysis is often based on stochastic discrete event models like Markov models or stochastic Petri nets. For complex dynamical systems with numerous components, analytical expressions of the steady state are tedious to work out because of the combinatory explosion with discrete models. Moreover, the convergence of stochastic estimators is slow. For these reasons, fluidification can be investigated to estimate the asymptotic behaviour of stochastic processes with timed continuous Petri nets. The contributions of this paper are to sum up some properties of the asymptotic mean marking and average throughputs of stochastic and timed continuous Petri nets, then to point out the limits of the fluidification in the context of the stochastic steady state approximation. To overcome these limitations, the new semantic and the condition for convergence is proposed: fluid Petri nets with Non Linear Timed Continuous Petri Net (NL-CPN).
\end{abstract}

Keywords: Fluidification, Stochastic Petri Nets, Continuous Petri Nets, Steady State, Reliability Analysis

\section{Introduction}

Reliability analysis is a major challenge to improve the safety of industrial processes. For complex dynamical systems with numerous interdependent components, such studies are mainly based on stochastic discrete event models like Markov models or stochastic Petri nets (SPNs) [8]. Such models are mathematically well founded and can be investigated in order to work out either analytical results or numerical simulations. But in case of large systems, the combinatory explosion limits their use. In this context, fluidification can be discussed as a relaxation method.

The aim of this paper is to discuss the estimation of the SPNs asymptotic mean markings by using fluid models and to introduce new semantics of Continuous Peti nets (CPNs) for that issue. The fluidification of discrete Petri nets has been investigated first for the analysis and performance evaluation of discrete event systems (DES) [3], [4], [11], then for control applications [12], [7]. Recently fluidification has been also introduced for SPNs [13], [14]. In this paper, the limits of the fluidification of SPNs with timed CPNs with constant firing rates and infinite server semantic are first discussed. The set of asymptotic mean markings (reached from an identical initial marking) are sometimes different for
SPNs and CPNs with same structures.

The paper has five sections. The section two introduces PNs, SPNs, CPNs. Then, the section three discusses the fluidification of SPNs according to CPNs. The section four introduces Non Linear Timed Continuous Petri Net (NL$\mathrm{CPN}$ ) is proposed and discussed. The last section gives some conclusions

\section{Stochastic and Continuous Petri Nets}

\subsection{Petri Nets (PN)}

A Petri net (PN) is defined as $<\mathrm{P}, \mathrm{T}$, WPR, WPO $>$ where $\mathrm{P}=\{\mathrm{Pi}\}$ is a set of $\mathrm{n}$ places and $\mathrm{T}=\left\{\mathrm{T}_{\mathrm{j}}\right\}$ is a set of $\mathrm{q}$ transitions. WPR $=\left(\mathrm{w}^{\mathrm{PR}_{\mathrm{ij}}}\right) \in\left(\mathrm{Z}^{+}\right)^{\mathrm{n} \times \mathrm{q}}$ is the pre-incidence matrix where $Z^{+}$is the set of non negative integer numbers $\left(w^{P R}{ }_{i j}\right.$ is the weight of arc from place $P_{i}$ to transition $\left.T_{j}\right)$ and $n$ $\mathrm{x} \mathrm{q}$ are the dimensions of matrix WPR. WPO $=\left(\mathrm{w}^{\mathrm{PO}}{ }_{\mathrm{ij}}\right)$ $\in\left(\mathrm{Z}^{+}\right)^{\mathrm{n} \times \mathrm{q}}$ is the post-incidence one $\left(\mathrm{w}^{\mathrm{PO}}{ }_{\mathrm{ij}}\right.$ is the weight of arc from transition $T_{j}$ to place $\left.P_{i}\right)$ [4]. The PN incidence matrix $W$ is defined as $\mathrm{W}=\mathrm{WPO}-\mathrm{WPR} \in(\mathrm{Z})^{\mathrm{n} \times \mathrm{q}}$. The PN marking vector $M(t)=\left(m_{i}(t)\right), i=1, \ldots . n$, is an application from the set of non negative real numbers $\mathrm{R}^{+}$to $\left(\mathrm{Z}^{+}\right)^{\mathrm{n}}$ such that, for each place $P_{i} \in P, m_{i}(t)=M\left(P_{i}, t\right)$ is the number of tokens in place $P_{i}$ at time t. $M_{I}$ is the PN initial marking and $M(t)=\left(m_{i}(t)\right)$, i 
$=1, \ldots \mathrm{n}$ is the mean marking vector at time $\mathrm{t}$ (i.e. $\mathrm{m}_{\mathrm{i}}(\mathrm{t})$ is the mean number of tokens in place $P_{i}$ during the time interval $[0, t])$. Each transition $T_{j}$ fires according to the round towards zeros (i.e. "floor" function) of its enabling degree $n_{j}(M(t)$ ) defined for marking $M(t)$ by equation (1):

$$
\mathrm{n}_{\mathrm{j}}(\mathrm{M})=\min \left(\mathrm{m}_{\mathrm{i}} / \mathrm{w}^{\mathrm{PR}}{ }_{\mathrm{ij}}\right) \text { for all } \mathrm{P}_{\mathrm{i}} \in{ }^{\circ} \mathrm{T}_{\mathrm{j}}
$$

where ${ }^{\circ} \mathrm{T}_{\mathrm{j}}$ stands for the set of $\mathrm{T}_{\mathrm{j}}$ upstream places. The place $P_{i}$ such that $i=\operatorname{argmin}\left(m_{k}(t) / w^{P R}{ }_{k j}\right)$ for all $P_{k} \in{ }^{\circ} T_{j}$ is the critical place for transition $T_{j}$ at time $t$. Right and left natural annullers of the token flow matrix $\mathrm{W}$ are called $\mathrm{T}$-semiflows and $\mathrm{P}$-semi flows, respectively. When $\mathrm{Y}^{\mathrm{T}} . \mathrm{W}=0$, the net is said to be conservative and when $\mathrm{W} . \mathrm{x}=0$. The net is said to be consistent. In a consistent net, a vector $\mathrm{x}$ such that $\mathrm{W} . \mathrm{x}=$ 0 represents a repetitive sequence, or in other words, a potential steady-state behaviour of the system in which all transitions are fired [17].

\subsection{Stochastic Petri Nets (SPN)}

A stochastic Petri net is defined as a timed PN whose transitions firing periods are characterized by random distributions. The basic model (SPN) has been introduced by [8], [9] and several extensions have been developed [1], [5] for the reliability analysis of reparable systems. Basically, a SPN, $<\mathrm{PN}, \mu>$, is a PN with a firing rate vector $\mu=\left(\mu_{j}\right) \in\left(R^{+}\right)^{q}$. Each transitions $T_{j}$ is characterized by the firing rate $\mu_{j}$ so that $\mu_{j}$. dt is the probability that the transition $T_{j}$ will fire between $t$ and $t+d t$ when the transition $T_{j}$ has been enabled, with degree 1 at $t$. The marking process of a SPN is characterized according to the PN incidence matrices, the initial marking, the firing rates, the firing policy, the server policy and the execution policy [2], [5]. The average throughput, marking and mean marking vectors of a SPN at time $t$ will be referred as $X_{s}(t), M_{s}(t)$. The SPNs that are considered in this paper satisfy the assumptions $\left(\mathrm{H}_{1}\right)$ to $\left(\mathrm{H}_{5}\right)$ :

$\left(\mathrm{H}_{1}\right)$ the marked SPNs are bounded.

$\left(\mathrm{H}_{2}\right)$ the marked SPNs are reinitialisable.

$\left(\mathrm{H}_{3}\right)$ the firing policy is a race policy: the transition whose firing time elapses first is assumed to be the one that will fire next.

$\left(\mathrm{H}_{4}\right)$ the server policy is of type infinite server: the minimal period of each transition $T_{j}$ is defined with a stochastic duration which is characterized according to an exponential distribution of varying parameter: floor $\left(n_{j}(M\right.$ (t))). $\mu_{\mathrm{j}}$.

$\left(\mathrm{H}_{5}\right)$ the execution policy is of type resampling memory: at the entrance in a marking, the remaining firing time of all transitions that were enabled is reset.

As a consequence, the considered SPNs have a reachability graph $\mathrm{R}\left(\mathrm{SPN}, \mathrm{M}_{\mathrm{I}}\right)$ with a finite number $\mathrm{N}$ of states and their marking process is mapped into a Markov model with state space isomorphic to $\mathrm{R}\left(\mathrm{SPN}, \mathrm{M}_{\mathrm{I}}\right)$ [8], [2]. In that case, the asymptotic behaviour of SPNs can be worked out according to the steady state probabilities of the Markov model.

\subsection{Continuous Petri Nets}

CPNs have been developed in order to provide continuous approximations of the discrete behaviours of PNs. The marking of each place is a continuous non negative real valued function of time [11]. A $\mathrm{CPN}$ is defined as $\angle \mathrm{PN}$, $\mathrm{X}_{\max }>$ where PN is a Petri nets and $\mathrm{X}_{\max }=\operatorname{diag}\left(\mathrm{x}_{\max }\right) \in$ $\left(R^{+}\right)^{\mathrm{qxq}}$ is the diagonal matrix of maximal firing speeds $x_{\operatorname{maxj}}, j$ $=1, \ldots \mathrm{q}[4] . \mathrm{M}_{\mathrm{c}}(\mathrm{t})$ is the marking vector and $\mathrm{X}_{\mathrm{c}}(\mathrm{t})=\left(\mathrm{x}_{\mathrm{cj}}(\mathrm{t})\right) \in$ $\left(\mathrm{R}^{+}\right)^{\mathrm{q}}$ is the firing speeds vector at time t. The marking variation is given by (2):

$$
\mathrm{dM}_{\mathrm{c}}(\mathrm{t}) / \mathrm{dt}=\mathrm{W} \cdot \mathrm{X}_{\mathrm{c}}(\mathrm{t}), \mathrm{M}_{\mathrm{c}}(0)=\mathrm{M}_{\mathrm{I}}
$$

Finite server (i.e. constant speeds) and infinite server (i.e. variable speeds) semantics exist for CPN. In this paper only the infinite server semantic is considered. $X_{c}(t)$ depends continuously on the marking of the places according to (3):

$$
\mathrm{x}_{\mathrm{cj}}(\mathrm{t})=\mathrm{x}_{\max j} \cdot \mathrm{n}_{\mathrm{j}}\left(\mathrm{M}_{\mathrm{c}}(\mathrm{t})\right)
$$

\subsection{Steady States of SPNS}

For live SPN that satisfy hypotheses $\left(\mathrm{H}_{1}\right)$ to $\left(\mathrm{H}_{5}\right)$ and with a finite number of states in the reachability graph, the marking process is mapped into a Markov model with state space isomorphic to the reachability graph of the SPN model [2]; [9]; [13] In that case the steady state of SPN can be worked out according to the steady state probabilities of the Markov model.

Let define $\mathrm{X}_{\mathrm{s}}=\left(\mathrm{x}_{\mathrm{sj}}\right) \in\left(\mathrm{R}^{+}\right)^{\mathrm{q}}$ as the average throughputs vector and $\mathrm{M}_{\mathrm{s}}=\left(\mathrm{m}_{\mathrm{si}}\right) \in\left(\mathrm{R}^{+}\right)^{\mathrm{n}}$ as the mean markings vector of SPN and $\Pi_{\mathrm{ss}}=\left(\pi_{\mathrm{ssk}}\right) \in[0,1]^{1 \times \mathrm{N}}$ as the steady state probabilities vector of the associated Markov model with $\mathrm{N}$ states. Let define $\mathrm{A}(\mu) \in(\mathrm{R})^{\mathrm{N} \times \mathrm{N}}$ as the generator of the underlying continuous time Markov model (i.e. the continuous time Markov model that defines the variations of the state probability vector according to the Petri net reachability graph) and $1_{\mathrm{N}}=(1, \ldots, 1)^{\mathrm{T}} \in\left(\mathrm{R}^{+}\right)^{\mathrm{N}}$ as the column vector of size $\mathrm{N}$ with all entries equal to 1 . It is well known that $\Pi_{s s}$ is the single solution of equations (4) [7], [10]:

$$
\begin{aligned}
& \Pi_{\mathrm{ss}} \cdot \mathrm{A}(\mu)=0 \\
& \Pi_{\mathrm{ss}} \cdot 1_{\mathrm{N}}=1
\end{aligned}
$$

As a consequence, $\mathrm{X}_{\mathrm{s}}$ and $\mathrm{M}_{\mathrm{s}}$ are obtained according to equations (5) and (6):

$$
\begin{gathered}
x_{s j}=\mu_{j} \cdot\left(\sum_{k=1 \ldots N} n_{j}\left(M_{k}\right) \cdot \pi_{k}\right) \\
m_{s i}=\sum_{k=1 \ldots N} m_{k i} \cdot \pi_{k}
\end{gathered}
$$

where $\mathrm{M}_{\mathrm{k}}=\left(\mathrm{m}_{\mathrm{ki}}\right) \in\left(\mathrm{R}^{+}\right)^{\mathrm{n}}$ stands for the marking vector corresponding to the state $\mathrm{k}$ of the Markov model. This method gives an analytical solution of the SPN steady state in case of ergodic Markov models, but requires the computation of the transition matrix $A(\mu)$ and as a consequence the reachability graph $\mathrm{R}\left(\mathrm{PN}, \mathrm{M}_{\mathrm{I}}\right)$ of the SPN. For large systems, the computational time and memory requirements necessary 
to work out $\mathrm{R}\left(\mathrm{PN}, \mathrm{M}_{\mathrm{I}}\right)$ become important as long as the number $\mathrm{N}$ of states increases exponentially. In that sense, SPN can be considered as a stochastic estimator for the Markov model. The advantage of this estimator is that the determination of $\mathrm{R}\left(\mathrm{PN}, \mathrm{M}_{\mathrm{I}}\right)$ is no longer required, the drawback is the slow convergence of the stochastic estimator, particularly in case of rare events.

The system in Figure 1 models a simple manufacturing system. The final product is composed of two different parts, $A$ and $B$, that are processed in machines $M_{1}$ and $M_{2}$ (represented by transitions $\mathrm{T}_{1}$ and $\mathrm{T}_{2}$ ), and stored in buffers $\mathrm{P}_{4}$ and $\mathrm{P}_{6}$, respectively. Then, they are assembled by $\mathrm{M}_{3}$ (i.e. transition $T_{3}$ ), and processed in $M_{4}$ (i.e. transition $T_{4}$ ).
Finally, $\mathrm{M}_{5}$ (i.e. transition $\mathrm{T}_{5}$ ) packages them. During the processing of parts $\mathrm{A}$ and $\mathrm{B}$, tooll (tokens in place $\mathrm{P}_{5}$ ) and tool2 (tokens in place $\mathrm{P}_{7}$ ) are needed. Also tool3 (tokens in place $\mathrm{P}_{3}$ ) has to be used in the three final operations. The machines $\mathrm{M}_{1}, \mathrm{M}_{2}, \mathrm{M}_{4}$ and $\mathrm{M}_{5}$ are assumed to be reliable and an active redundancy $(n=3)$ is considered for the assembly machine $\mathrm{M}_{3}$ that is assumed to have failure and repair rates $\lambda$ $=1.5 \mathrm{e}^{-2} \mathrm{TU}^{-1}$ and $\mu=1 \mathrm{e}^{-1} \mathrm{TU}^{-1}$. To achieve high availability requirements 3 active redundancies are considered for $\mathrm{M}_{3}$ (place $\mathrm{P}_{10}$ ). The productivity of the workshop is evaluated with the computation of the output flow $\mathrm{x}_{5}$ with respect to the

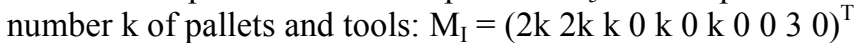

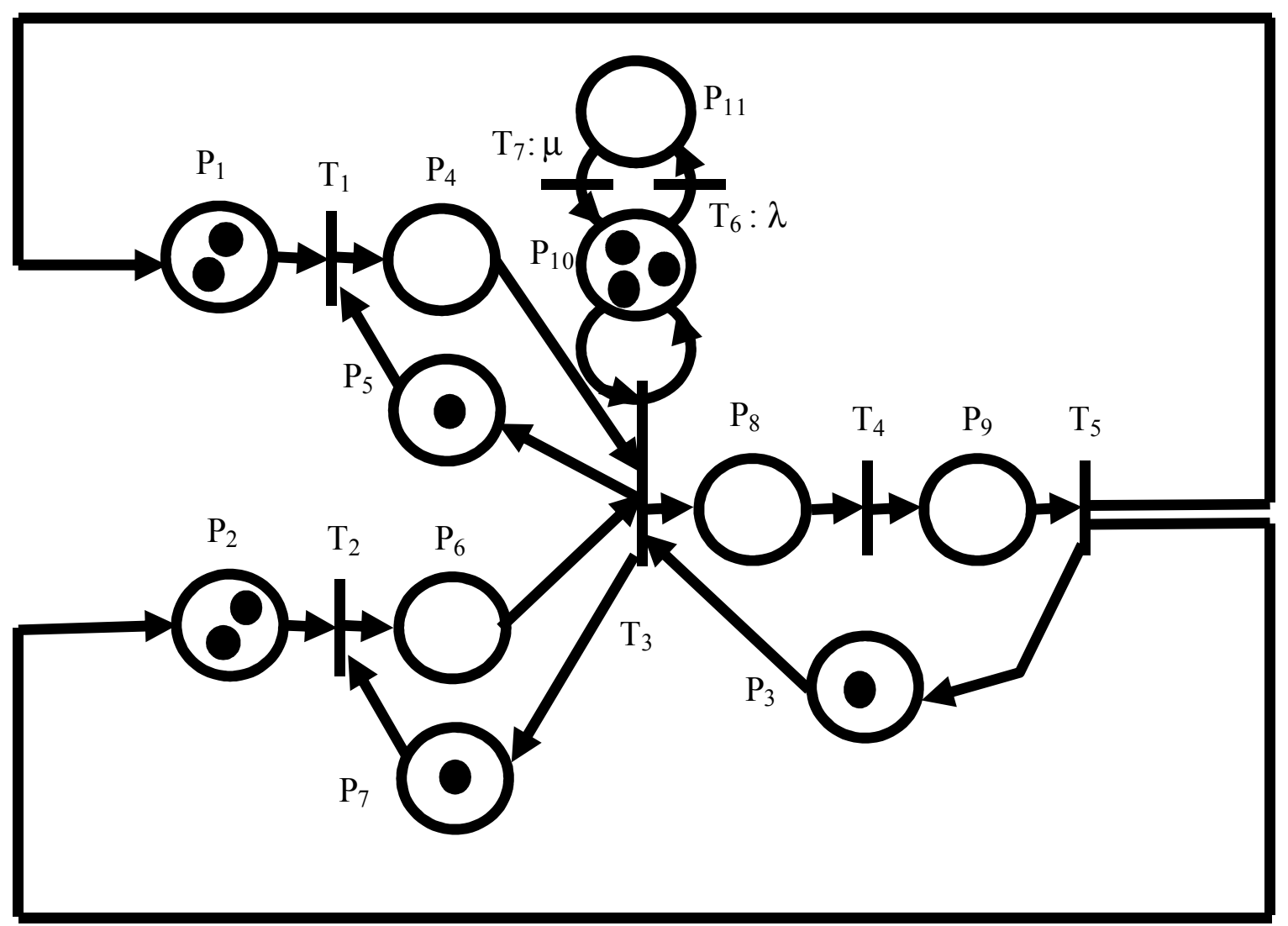

Figure 1. Assembly workshop.

Only the flow of transition $\mathrm{T}_{5}$ and the marking of places $\mathrm{P}_{1}, \mathrm{P}_{2}, \mathrm{P}_{8}$ and $\mathrm{P}_{9}$ will be considered. Other flows and markings are given by (7):

$$
\begin{aligned}
& \mathrm{x}_{1}=\mathrm{x}_{2}=\mathrm{x}_{3}=\mathrm{x}_{4}=2 \cdot \mathrm{x}_{5} \\
& \mathrm{~m}_{4}=\mathrm{y}_{4} \cdot \mathrm{M}_{\mathrm{I}}-\mathrm{m}_{1}-\mathrm{m}_{8}-\mathrm{m}_{9} \\
& \mathrm{~m}_{6}=\mathrm{y}_{5} \cdot \mathrm{M}_{\mathrm{I}}-\mathrm{m}_{2}-\mathrm{m}_{8}-\mathrm{m}_{9} \\
& \mathrm{~m}_{3}=\mathrm{y}_{3} \cdot \mathrm{M}_{\mathrm{I}}-\mathrm{m}_{8}-\mathrm{m}_{9} \\
& \mathrm{~m}_{5}=\mathrm{y}_{1} \cdot \mathrm{M}_{\mathrm{I}}-\mathrm{y}_{4} \cdot \mathrm{M}_{\mathrm{I}}+\mathrm{m}_{1}+\mathrm{m}_{8}+\mathrm{m}_{9} \\
& \mathrm{~m}_{7}=\mathrm{y}_{2} \cdot \mathrm{M}_{\mathrm{I}}-\mathrm{y}_{5} \cdot \mathrm{M}_{\mathrm{I}}+\mathrm{m}_{2}+\mathrm{m}_{8}+\mathrm{m}_{9}
\end{aligned}
$$

Equations (7) hold for SPN and CPN. Steady state markings and flows are reported in Table 1 with respect to initial marking.

Table 1. Number of states, average throughputs and mean markings of SPN from Figure 1 in function of $k$.

\begin{tabular}{lllllll}
\hline $\mathbf{k}$ & $\mathbf{N}$ & $\mathbf{X}_{\mathbf{s} \mathbf{5}}$ & $\mathbf{m}_{\mathbf{s} 1}$ & $\mathbf{m}_{\mathbf{s} 2}$ & $\mathbf{m}_{\mathbf{s} \mathbf{8}}$ & $\mathbf{m}_{\mathbf{s} \mathbf{9}}$ \\
\hline 1 & 205 & 0.50 & 1.75 & 1.75 & 1.00 & 1.48 \\
2 & 1885 & 1.13 & 3.51 & 3.51 & 2.25 & 2.75 \\
3 & 7796 & 1.77 & 5.20 & 5.21 & 3.54 & 4.07 \\
4 & 22187 & 2.42 & 6.97 & 6.91 & 4.84 & 5.36 \\
$\ldots$ & $\ldots$ & $\ldots$ & $\ldots$ & $\ldots$ & $\ldots$ & $\ldots$ \\
$\infty$ & $\infty$ & $\infty$ & $\infty$ & $\infty$ & $\infty$ & $\infty$ \\
\hline
\end{tabular}

The productivity of the system in Figure 1 is evaluated with the computation of the output flow $\mathrm{x}_{\mathrm{s} 5}(\mathrm{t})$ with respect to the number of pallets and tools. The results obtained with Markov models and SPNs simulation over a time interval of $\mathrm{D}=1000 \mathrm{TU}$ are summed up in Table 2 and Table 3. 
Table 2. Performance evaluation with Markov models.

\begin{tabular}{llll}
\hline $\mathbf{k}$ & $\mathbf{N}$ & $\mathbf{x}_{\mathbf{5}}(\mathbf{t})$ Markov model & Computational effort $(\mathbf{T U})$ \\
\hline 1 & 48 & 0.29 & 0.1 \\
2 & 216 & 0.61 & 0.9 \\
3 & 640 & 0.93 & 12 \\
4 & 1500 & 1.25 & 108 \\
$\ldots$ & $\ldots$ & $\ldots$ & $\ldots$ \\
\hline
\end{tabular}

For $\mathrm{k}>4$, the computational effort becomes heavy because of the large number $\mathrm{N}$ of states and the performance evaluation with Markov model analysis is no longer computable [17].

Table 3. Performance evaluation with SPNs.

\begin{tabular}{lll}
\hline $\mathbf{k}$ & $\mathbf{x}_{\mathbf{s} 5}(\mathbf{t}) \mathbf{S P N}$ & Computational effort $(\mathbf{T U})$ \\
\hline 1 & 0.30 & 0.59 \\
2 & 0.61 & 2.0 \\
3 & 0.94 & 4.0 \\
4 & 1.24 & 6.8 \\
5 & 1.55 & 11 \\
10 & 2.41 & 30 \\
\hline
\end{tabular}

Simulation with SPN can be used to overcome the computational limitation with Markov model. The simulation error does not exceed $1 \%$. One can also notice that the computational effort increases but remains acceptable up to $\mathrm{k}=10$. For $\mathrm{k}>10$, fluidification must be introduced.

\section{Fluidification of SPN}

The aim of this section is to pinpoint the limit of the fluidification of SPNs by CPNs with same structure and same initial marking. The several example for which CPNs do not converge to the asymptotic mean marking of SPNs is presented.

\subsection{Trivial Fluidification of SPNs}

The steady state of a SPN $=<\mathrm{PN}, \mu>$ and the one resulting of a $\mathrm{CPN}=<\mathrm{PN}, \mathrm{X}_{\max }>$ with same structure, initial marking and $\mathrm{X}_{\max }=\mu$ are in general case not identical. For example, the steady state of the PN in Figure 1 considered as continuous is given in Table 4. It can also be noticed that the difference between SPN and CPN steady state decreases with respect to the marking. More generally, a PN can be live as discrete and non-live as continuous. In a similar way, a discrete bounded system may be unbounded as continuous [11]. Moreover, like in discrete nets, the throughput of a CPN does not fulfil in general any monotonicity property, neither with respect to the initial marking, nor with respect to the structure of the net, nor with respect to the transitions rates. [12]. The throughput of a CPN is notin general an upper bound of the throughput of the discrete PN [6].

Table 4. Average throughputs and mean markings of CPN from Figure 1 in function of $k$.

\begin{tabular}{llllll}
\hline $\mathbf{k}$ & $\mathbf{x}_{\mathbf{c} 5}$ & $\mathbf{m}_{\mathbf{c 1}}$ & $\mathbf{m}_{\mathbf{c} 2}$ & $\mathbf{m}_{\mathbf{c} 8}$ & $\mathbf{m}_{\mathbf{c} 9}$ \\
\hline 1 & 0.67 & 1.67 & 1.67 & 1.33 & 1.33 \\
2 & 1.33 & 3.33 & 3.33 & 2.67 & 2.67 \\
3 & 2 & 5 & 5 & 4 & 4 \\
4 & 2.67 & 6.67 & 6.67 & 5.33 & 5.33 \\
$\ldots$ & $\ldots$ & $\ldots$ & $\ldots$ & $\ldots$ & $\ldots$ \\
$\infty$ & $\infty$ & $\infty$ & $\infty$ & $\infty$ & $\infty$ \\
\hline
\end{tabular}

\subsection{Regions in the Reachability Space}

According to the function "min (.)" in the expression of the enabling degree (1), the marking space of CPNs is divided into $\mathrm{K}$ regions $\mathrm{A}_{\mathrm{k}}$ with $\mathrm{K}=\left|{ }^{\circ} \mathrm{T}_{1}\right| \ldots \ldots{ }^{\circ} \mathrm{T}_{\mathrm{q}} \mid$. Each region $A_{k}$ is defined by its configuration [7], also called PTset [6] defined by (8):

$$
\begin{gathered}
\text { PT-set }\left(\mathrm{A}_{\mathrm{k}}\right)=\left\{\left(\mathrm{P}_{\mathrm{i}}, \mathrm{T}_{\mathrm{j}}\right)\right. \text { s. t. } \\
\left.\forall \mathrm{M}_{\mathrm{c}}(\mathrm{t}) \in \mathrm{A}_{\mathrm{k}}, \mathrm{x}_{\mathrm{cj}}(\mathrm{t})=\mathrm{x}_{\text {maxj }}(\mathrm{t}) \cdot \mathrm{m}_{\mathrm{ci}}(\mathrm{t}) / \mathrm{w}^{\mathrm{PR}}{ }_{\mathrm{ij}}\right\}
\end{gathered}
$$

The place $P_{i}$ such that $i=\operatorname{argmin}\left(m_{k}(t) / w^{P R}{ }_{k j}\right)$ for all $P_{k}$ $\in{ }^{\circ} T_{j}$ is the critical place for transition $T_{j}$ at time t. PT$\operatorname{set}\left(A_{k}\right)$ represents the set of all pairs $\left(P_{i}, T_{j}\right), j=1, \ldots$, q such that $P_{i}$ is the critical place of transition $T_{j}$ in region $A_{k}$.

According to the PT-sets. A region $\mathrm{A}_{\mathrm{k}}$ is critical if there exists two transitions $T_{j}$ and $T_{m}$ that have the same critical place $P_{i}$ in region $A_{k}$ (i.e. $\left(P i, T_{j}\right) \in P T$-set $\left(A_{k}\right)$ and $\left(P_{i}, T_{m}\right)$ $\in$ PT-set $\left(A_{k}\right)$ ). In a critical region $A_{k}$, a single place drives the throughputs of at least two distinct transitions and the rank of matrix $A_{k}$ is less than the rank of the constraint matrices in other regions.

Each r-region $A_{k}$ is characterized by a constraint matrix $A_{k}$ $=\left(a^{k}{ }_{i j}\right) \in\left(R^{+}\right)^{q \times n}, k=1, \ldots, K, i=1, \ldots, q$ and $j=1, \ldots, n$ :

- $\mathrm{a}_{\mathrm{ji}(\mathrm{k}, \mathrm{j})}=1 / \mathrm{w}^{\mathrm{PR}}{ }_{\mathrm{i}(\mathrm{k}, \mathrm{j}) \mathrm{j}}$ for all $\mathrm{T}_{\mathrm{j}} \in \mathrm{T}$,

- $\mathrm{a}_{\mathrm{ji}}^{\mathrm{k}}=0$ otherwise.

As a consequence, in each region $A_{k}$, the firing speed vector can be written as $X_{c}(t)=X_{\max } \cdot A_{k} \cdot M_{c}(t)$, equation (9) holds:

$$
\forall \mathrm{M}_{\mathrm{c}}(\mathrm{t}) \in \mathrm{A}_{\mathrm{k}}, \mathrm{dM}_{\mathrm{c}}(\mathrm{t}) / \mathrm{dt}=\mathrm{W} \cdot \mathrm{X}_{\max } \cdot \mathrm{A}_{\mathrm{k}} \cdot \mathrm{M}_{\mathrm{c}}(\mathrm{t})
$$

\subsection{Problem Statement}

Fluidification of discrete PNs leads to some unexpected results and numerous structural and behavioural properties are not preserved with fluidification. In particular, a PN can be live as discrete and non-live as continuous and a discrete bounded system may be unbounded as continuous [12]. The throughput of a CPN is mainly not identical to the throughput of a discrete PN. It is often but not always an upper bound of this throughput [6], [18].

Concerning SPNs, the approximation of the steady state by CPNs is of particular interest as long as the estimation of the asymptotic mean markings can be used to work out the availability, the mean time between failures, and other indicators for reliability. Large approximation errors appear frequently even if such errors decrease with respect to the initial marking. The asymptotic mean markings of SPNs can be approximated (with an acceptable accuracy) with the steady state of CPNs if (1) all transitions remain enabled with degree at least 1 in long run; (2) the marking vector does not leave the region of initial marking in long run. Markovian Continuous Petri Nets (i.e. CPNs with an additive normally distributed noise) have been investigated to relaxe the condition (2) [13]. In addition, a partial relaxation of SPNs leads to Hybrid Markovian Continuous Petri Nets and can be used to relax condition (1) [14], [19], [21]. But in the former works the continuous models are non-deterministic and the steady state is no longer easy to work out. 


\section{Fluidification with NL-CPN}

In this section, continuous Petri nets with adaptive maximal firing speeds are proposed to converge to the asymptotic mean marking of SPNs also in critical regions [18]. The maximal firing speeds are considered as functions of time and updated in order to compensate on line the errors on the throughputs and markings regarding the asymptotic stochastic average throughputs and mean markings to be reached. Non linear timed continuous Petri nets with adaptive maximal firing speeds are defined as a consequence.

\subsection{Definition}

A Non Linear Timed Continuous Petri Net: NL- CPN $=$ $<$ P, T, WPR, WPO, Ms, Xs $>$ is defined by a set of $n+q$ differential equations defined by (10):

$$
\mathrm{dM}_{\mathrm{c}}(\mathrm{t}) / \mathrm{dt}=\mathrm{W} \cdot \mathrm{X}_{\mathrm{c}}(\mathrm{t})
$$

$\mathrm{dX}_{\max } / \mathrm{dt}=\eta \cdot \operatorname{diag}\left(\beta \cdot \mathrm{W}^{\mathrm{T}} \cdot\left(\mathrm{M}_{\mathrm{s}}-\mathrm{M}_{\mathrm{c}}(\mathrm{t})\right)+(1-\beta) \cdot\left(\mathrm{X}_{\mathrm{s}}-\mathrm{X}_{\mathrm{c}}(\mathrm{t})\right)\right)$

under the constraint $X_{\max } \geq 0$.

$\eta$ is the adaptation parameter, and $\beta \in[0,1]$ is a ratio that balances the weight of the marking and throughput errors in the variation of $X_{\text {maxj }}$. The initial marking is $M_{I}$ and the initial maximal firing speeds are given by $\mathrm{X}_{\max }(0)=\operatorname{diag}(\mu)$. In order to motivate the updating equation (10) of the maximal firing speeds, let consider a transition $\mathrm{T}_{\mathrm{j}}$ and define $\mathrm{w}_{\mathrm{j}}$ as the column $j$ of incidence matrix $W$. If at time $t$, for any places $P_{i} \in{ }^{\circ} T_{j}$, the marking $\mathrm{m}_{\mathrm{ci}}(\mathrm{t})$ satisfies $\mathrm{m}_{\mathrm{si}}-\mathrm{m}_{\mathrm{ci}}(\mathrm{t})>0\left(\right.$ resp. $\left.\mathrm{m}_{\mathrm{si}}-\mathrm{m}_{\mathrm{ci}}(\mathrm{t})<0\right)$ and for any places $P_{k} \in T_{j}^{\circ}$, the marking $m_{c k}(t)$ satisfies $m_{s k}-$ $\mathrm{m}_{\mathrm{ck}}(\mathrm{t})<0\left(\right.$ resp. $\left.\mathrm{m}_{\mathrm{sk}}-\mathrm{m}_{\mathrm{ck}}(\mathrm{t})>0\right)$ then $\mathrm{w}_{\mathrm{j}}^{\mathrm{T}}\left(\mathrm{M}_{\mathrm{s}}-\mathrm{Mc}(\mathrm{t})\right)<0$ $\left(\right.$ resp. $\mathrm{w}_{\mathrm{j}}^{\mathrm{T}}\left(\mathrm{M}_{\mathrm{s}}-\mathrm{M}_{\mathrm{c}}(\mathrm{t})\right)>0$ ) and $\mathrm{x}_{\operatorname{maxj}}$ decreases (resp. $\mathrm{x}_{\operatorname{maxj}}$ increases). The variation of $\mathrm{X}_{\max }$ is also driven according to the firing speed $\mathrm{x}_{\mathrm{cj}}(\mathrm{t})$ of transition $\mathrm{T}_{\mathrm{j}}$ : If $\mathrm{x}_{\mathrm{sj}}-\mathrm{x}_{\mathrm{cj}}(\mathrm{t})<0$ (resp. $\mathrm{x}_{\mathrm{sj}}$ $\mathrm{x}_{\mathrm{cj}}(\mathrm{t})>0$ ) then $\mathrm{x}_{\operatorname{maxj}}$ decreases (resp. $\mathrm{x}_{\operatorname{maxj}}$ increases). But when the preceding conditions are not simultaneously satisfied, the convergence of $M_{c}(t)$ to $M_{s}$ is no longer monotonic.

\subsection{Introductive Example}

Consider for example the SPN described in Figure 2 [6]. This $\mathrm{PN}$ has $1 \mathrm{~T}$-semiflow $\mathrm{x}=\left(\begin{array}{llll}1 & 1 & 1 & 1\end{array}\right)^{\mathrm{T}}$ and $2 \mathrm{P}$-semiflows $\mathrm{y}_{1}=\left(\begin{array}{lllll}0 & 0 & 0 & 1 & 1\end{array}\right), \mathrm{y}_{2}=\left(\begin{array}{lllll}1 & 1 & 2 & 1 & 0\end{array}\right)$. As a consequence only the flow of transition $\mathrm{T}_{1}$ and the marking of places $\mathrm{P}_{1}, \mathrm{P}_{2}$ and $\mathrm{P}_{4}$ will be considered.

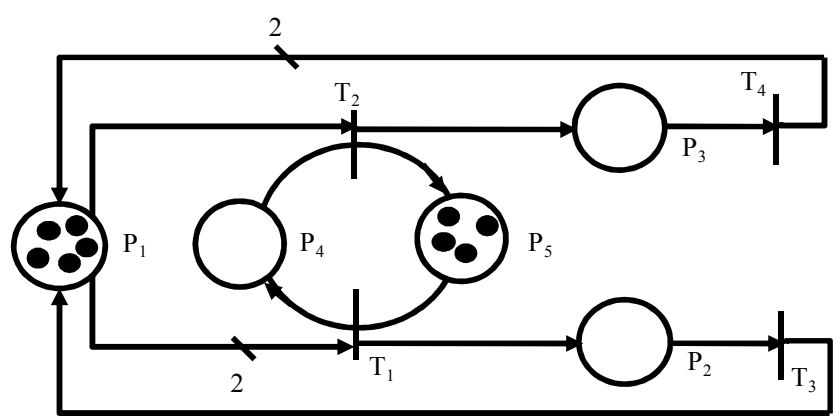

Figure 2. An SPN with firing rate vector $\mu=\left(\begin{array}{llll}3 & 1 & 1 & 10\end{array}\right)^{T}$ and initial marking $M_{I}=\left(\begin{array}{lllll}5 & 0 & 0 & 0 & 4\end{array}\right)^{T}$.
Throughput of $T_{1}(C P N$ : solid line, SPN: dashed line)

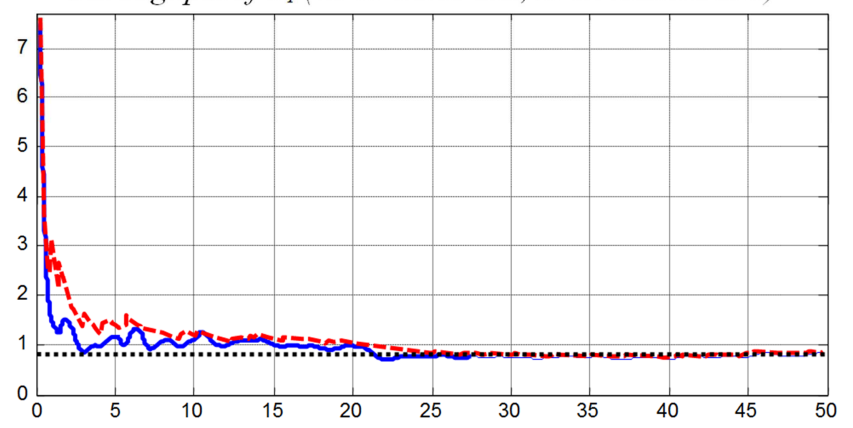

Figure 3. Throughput evolution of CPN (solid line) and SPN (dashed line) in function of time of Figure 2.
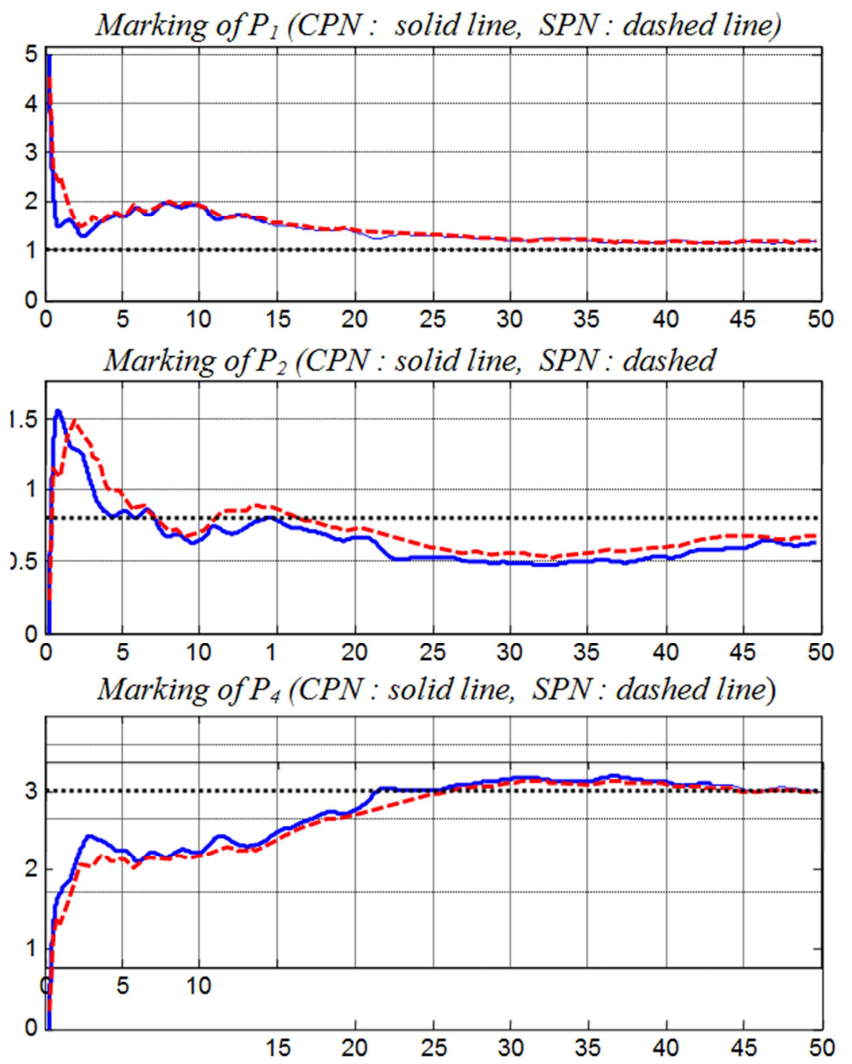

Figure 4. Markings evolution of CPN (solid line) and SPN (dashed line) in function of time of Figure 2.

under the constraint $X_{\max } \geq 0$, where $\eta$ is the adaptation parameter arbitrary fixed to $0.1, \operatorname{diag}(\mu) \in\left(\mathrm{R}^{+}\right)^{\mathrm{q} \times \mathrm{q}}$ is the diagonal matrix with SPN firing rates. As a consequence only the flow of transition $\mathrm{T}_{1}$ and the marking of places $\mathrm{P}_{1}, \mathrm{P}_{2}$ and $\mathrm{P}_{4}$ will be considered (Figure 3 and Figure 4).

\section{Conclusions}

This paper has pointed out that the asymptotic behaviour of SPNs cannot be trivially approximated with CPNs. Moreover it is not always possible to find any CPN with same structure and same initial marking that converges to the asymptotic mean marking of SPN in long run. In the present form, the proposed semantic cannot be used immediately for the performances evaluation of SPNs but provide an 
innovative point of view for the investigations of stochastic systems and may be used for controllers. Our future works are to continue the study of CPN. We will also study continuous approximations directly derived from the SPNs transition firing rates.

\section{References}

[1] Ajmone Marsa M., Chiola G. On Petri nets with deterministic and exponentially distributed firing times, Advances in Petri nets (Rozenberg G.), Springer Verlag, pp. 132-145, 1987.

[2] Bobbio A., Puliafito A., Telek M., Trivedi K. Recent Developments in Stochastic Petri Nets, Journal of Circuits, Systems, and Computers, Vol. 8, no. 1, Feb., pp. 119-158, 1998.

[3] Campos J., Chiola G., Silva M Ergodicity and throughut bounds of Petri nets with unique consistent firing count vector, IEEE Transactions on Software Engineering, Vol. 17, no 2, pp. 117-125, 1991.

[4] David R., Alla H., Petri nets and grafcet - tools for modelling discrete events systems, Prentice Hall, London, 1992.

[5] Diaz M., (Les réseaux de Petri: modèles fondamentaux, Hermes, Paris, 2001.

[6] Júlvez G., Recalde L. Silva M. Steady-state performance evaluation of continuous mono-T- semiflow Petri nets, Automatica, Vol. 41, no. 4, pp. 605-616, 2005.

[7] Mahulea C., Ramirez Trevino A., Recalde L., Silva M., Steady state control reference and token conservation laws in continuous Petri nets, Trans. IEEE - TASE, Vol. 5, no. 2, pp. 307-320, 2008.

[8] Molloy M. K. On the integration of delay and troughput in distributed processing models, Ph. D, UCLA, Los Angeles, USA, 1981.

[9] Molloy M. K., Performance analysis using stochastic Petri nets, IEEE Transactions on Computers C, vol. 31, pp. 913 $917,1982$.

[10] Rausand M. and Hoyland A. System reliability theory: models, statistical methods, and applications, Wiley, Hoboken, New Jersey, 2004.

[11] Silva M. and Recalde L. Petri nets and integrality relaxations: a view of continuous Petri nets, Trans. IEEE - SMC, part C, Vol. 32, no. 4, pp. 314-326, 2002.

[12] Silva M. and Recalde L. On fluidification of Petri Nets: from discrete to hybrid and continuous models, Annual Reviews in Control, Vol. 28, no. 2, pp. 253-266, 2004.

[13] Vazquez R., Recalde L., Silva M., Stochastic continuous-state approximation of markovian Petri net systems, Proceeding IEEE - CDC08, pp. 901-906, Cancun, Mexico, 2008.

[14] Vazquez R., Silva M., (Hybrid Approximations of Markovian Petri Nets, Proceeding IFAC - ADHS, pp. 56-61, Zaragoza, Spain, 2009.

[15] Lefebvre D, E. Leclercq, L. Khalij, E. Souza de Cursi, N. El Akchioui, Approximation of MTS stochastic Petri nets steady state by means of continuous Petri nets: a numerical approach, Proc. IFAC ADHS, pp. 62-67, Zaragoza, Spain, 2009.

[16] Lefebvre D, E. Leclercq, Piecewise constant timed continuous PNs for the steady state estimation of stochastic PNs, DISC, DOI: 10.1007/s10626-011-0114-y, 2011.

[17] Lefebvre D, E. Leclercq, N. El Akchioui, L. Khalij, E. Souza de Cursi, A geometric approach for the homothetic approximation of stochastic Petri nets, Proc. IFAC WODES, Berlin, Germany, 2010.

[18] Lefebvre D, About the stochastic and continuous Petri nets equivalence in long run, Non-Linear Analysis, Hybrid Systems (NAHS), vol. 5, pp. 394-406, 2011.

[19] Lei, K. Xing, L. Han, F. Xiong, Z. Ge, Deadlock-free scheduling for flexible manufacturing systems using Petri nets and heuristic search, Computers \& Industrial Engineering, vol. 72, pp. 297-305, 2014.

[20] Lefebvre. D, E. Leclercq, Control design for trajectory tracking with untimed Petri nets, accepted in IEEE Trans. Aut. Contr., to appear June 2015.

[21] Lefebvre. D and E. Leclercq, An algorithm based on model predictive control for trajectories tracking with untimed PNs, Proc. IEEE - MED 2014, pp. 243-248, Palermo, Italy, 2014. 